\title{
Supplements
}

Supplement equations:

gut:

$$
\frac{d A(1)}{d t}=-K_{a} \times A(1)
$$

central:

$$
\frac{d A(2)}{d t}=K_{a} \times A(1)-\frac{C L}{V c} \times A(2)-\frac{Q}{V c} \times A(2)+\frac{Q}{V p} \times A(3)
$$

peripheral:

$$
\frac{d A(3)}{d t}=\frac{Q}{V c} \times A(2)-\frac{Q}{V p} \times A(3)
$$

dGTP:

$$
\frac{d A(7)}{d t}=K_{\text {in }}^{0} \times\left(1-\frac{1}{1+\operatorname{Time}^{\gamma}} \times \frac{A(4)}{A(4)+E C_{50}}\right)-A(7) \times K_{\text {out }}
$$




\begin{tabular}{cccc}
\hline & $\begin{array}{c}\text { Model Output } \\
\text { Condition Number }\end{array}$ & $\begin{array}{c}\text { Bootstrapping } \\
\text { Success Rate }\end{array}$ & $\begin{array}{c}\text { VPC } \\
\text { Obs outside } 90 \% \mathrm{Cl}\end{array}$ \\
\hline TFV plasma & 25.3 & $99.2 \%$ & $7.59 \%$ \\
FTC plasma & 79.6 & $94.3 \%$ & $7.28 \%$ \\
TFV-DP IC & 24.9 & $99.3 \%$ & $1.81 \%$ \\
FTC-TP IC & 27.8 & $99.5 \%$ & $5.00 \%$ \\
dATP & 6.0 & $95.7 \%$ & $9.89 \%$ \\
dCTP & 5.5 & $99.7 \%$ & $10.4 \%$ \\
dGTP & 4.4 & $99.2 \%$ & $12.0 \%$ \\
TTP & 7.5 & $99.9 \%$ & $11.6 \%$ \\
\hline
\end{tabular}

Table A. Other model evaluation results. Obs: observed values. 

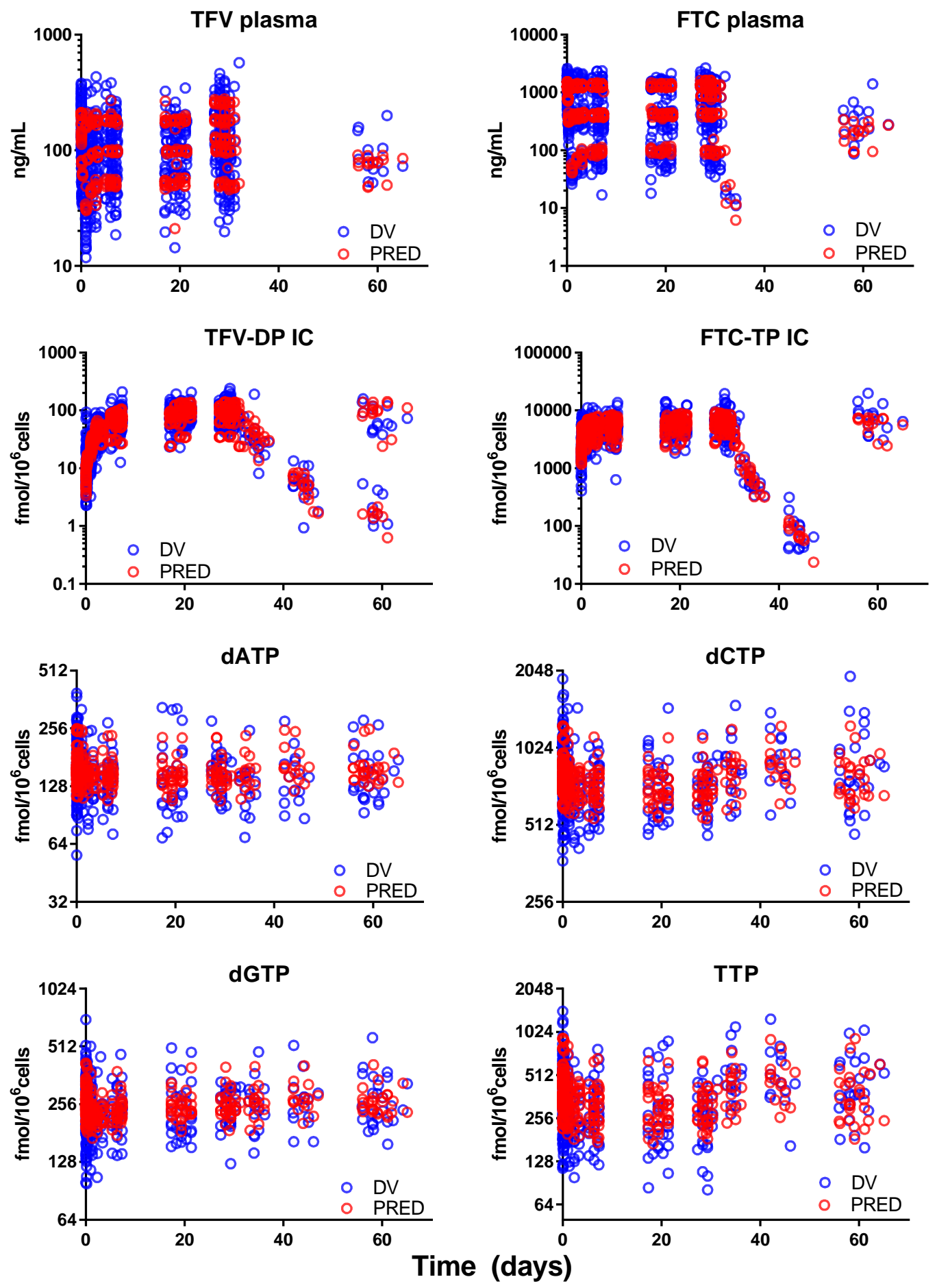

Figure A. Dependent variables (observed values) and predicted values vs time plots. IC: intracellular. PRED: predicted values. DV: dependent variables. 

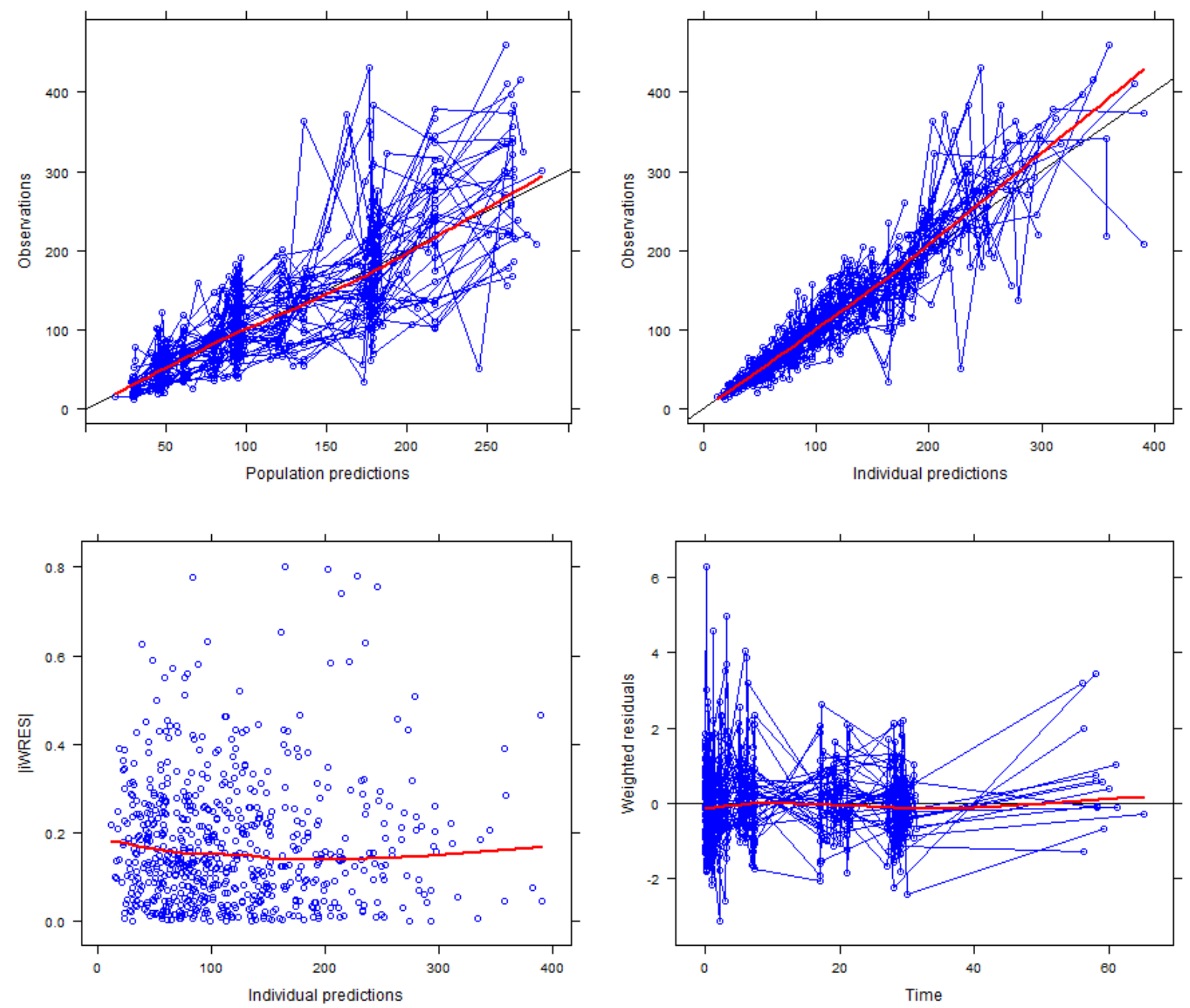

Figure B. Basic goodness of fit plots of TFV plasma model. Red line represents average values.

Black line represents theoretical values. Data from the same individual are showed in blue circles and are connected by lines. |iWRES|: absolute values of individual weighted residuals. 

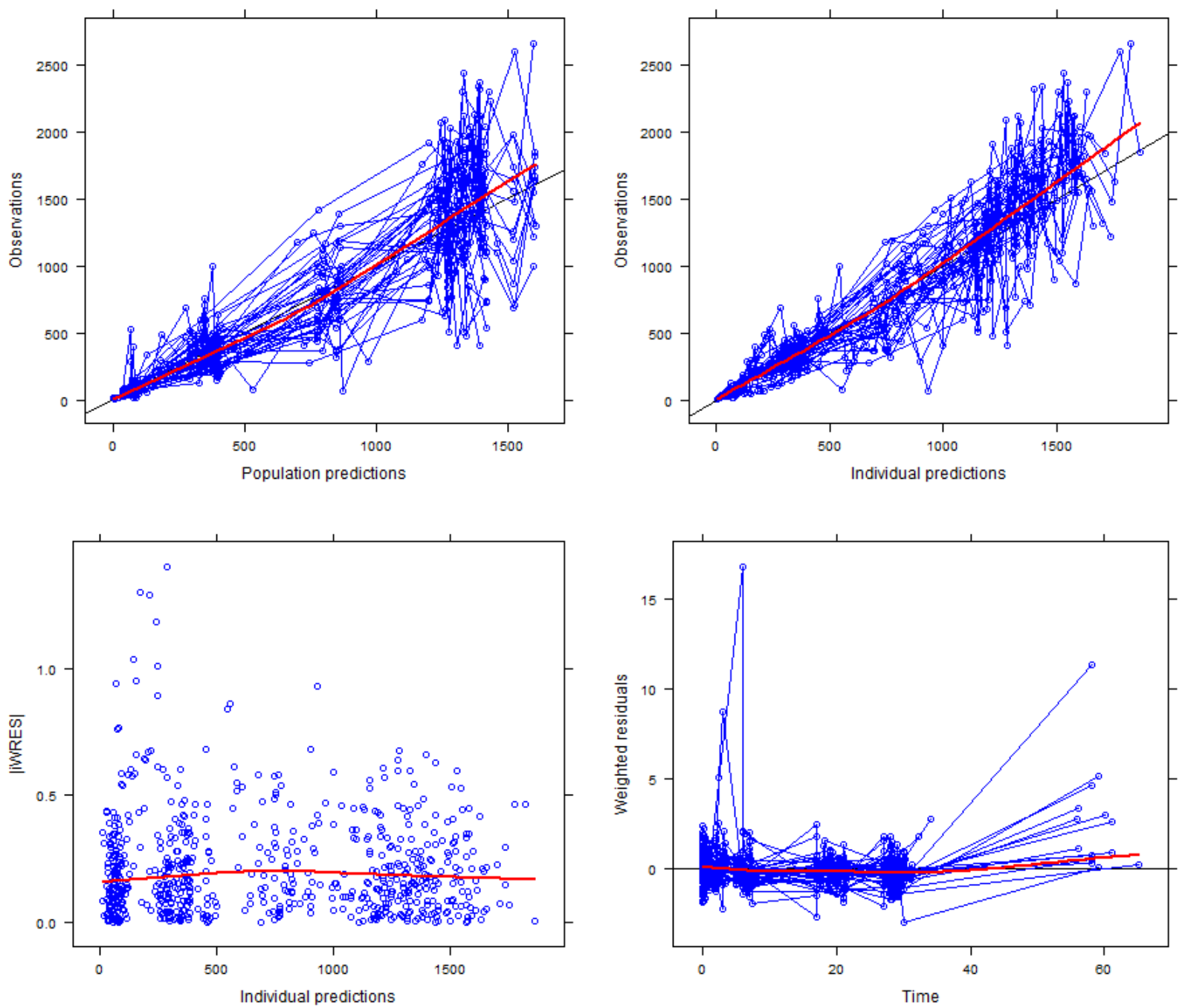

Figure C. Basic goodness of fit plots of FTC plasma model. Red line represents average values.

Black line represents theoretical values. Data from the same individual are showed in blue circles and are connected by lines. |iWRES|: absolute values of individual weighted residuals. 

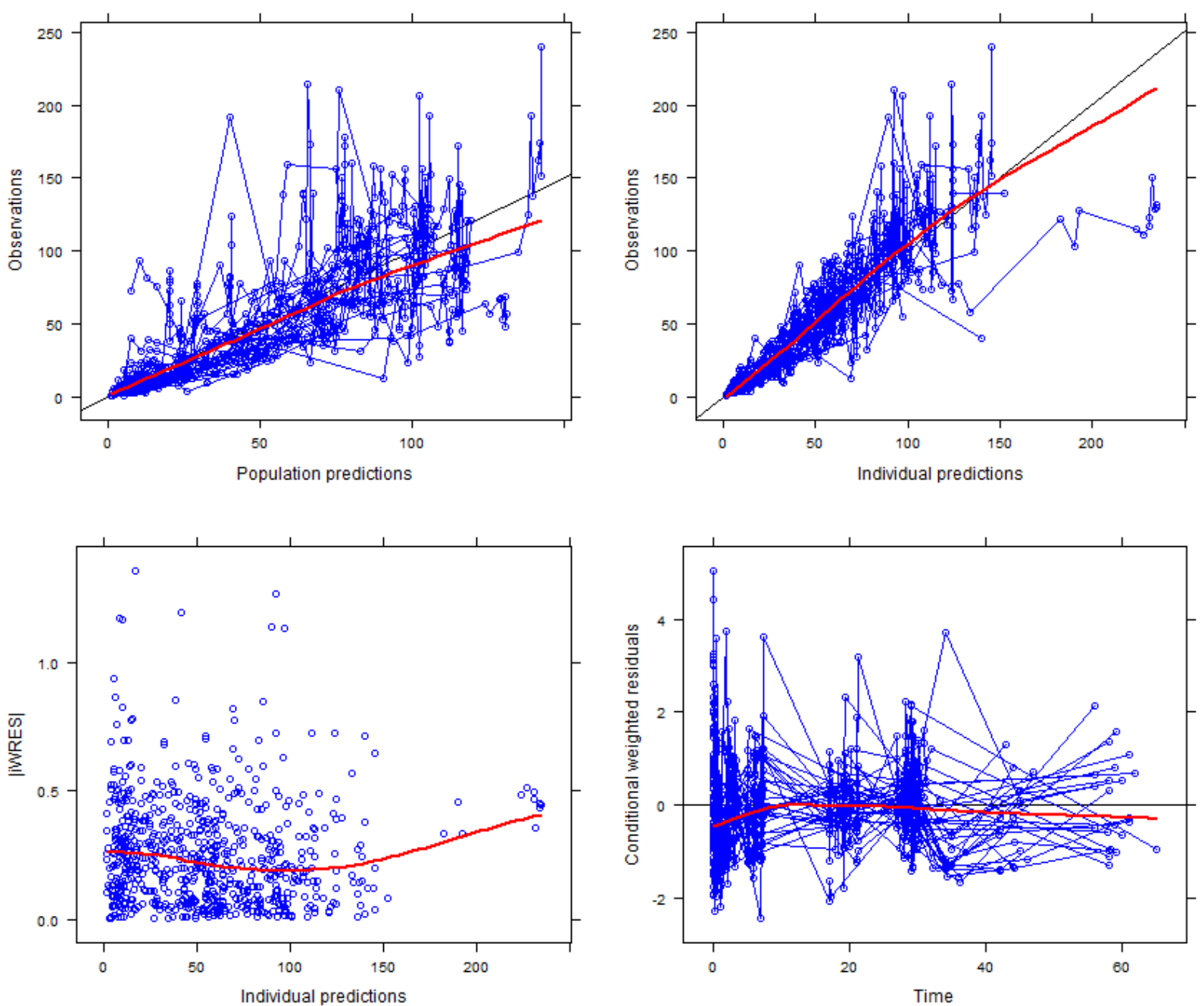

Figure D. Basic goodness of fit plots of intracellular TFV-DP model. Red line represents average values. Black line represents theoretical values. Data from the same individual are showed in blue circles and are connected by lines. |iWRES|: absolute values of individual weighted residuals. 

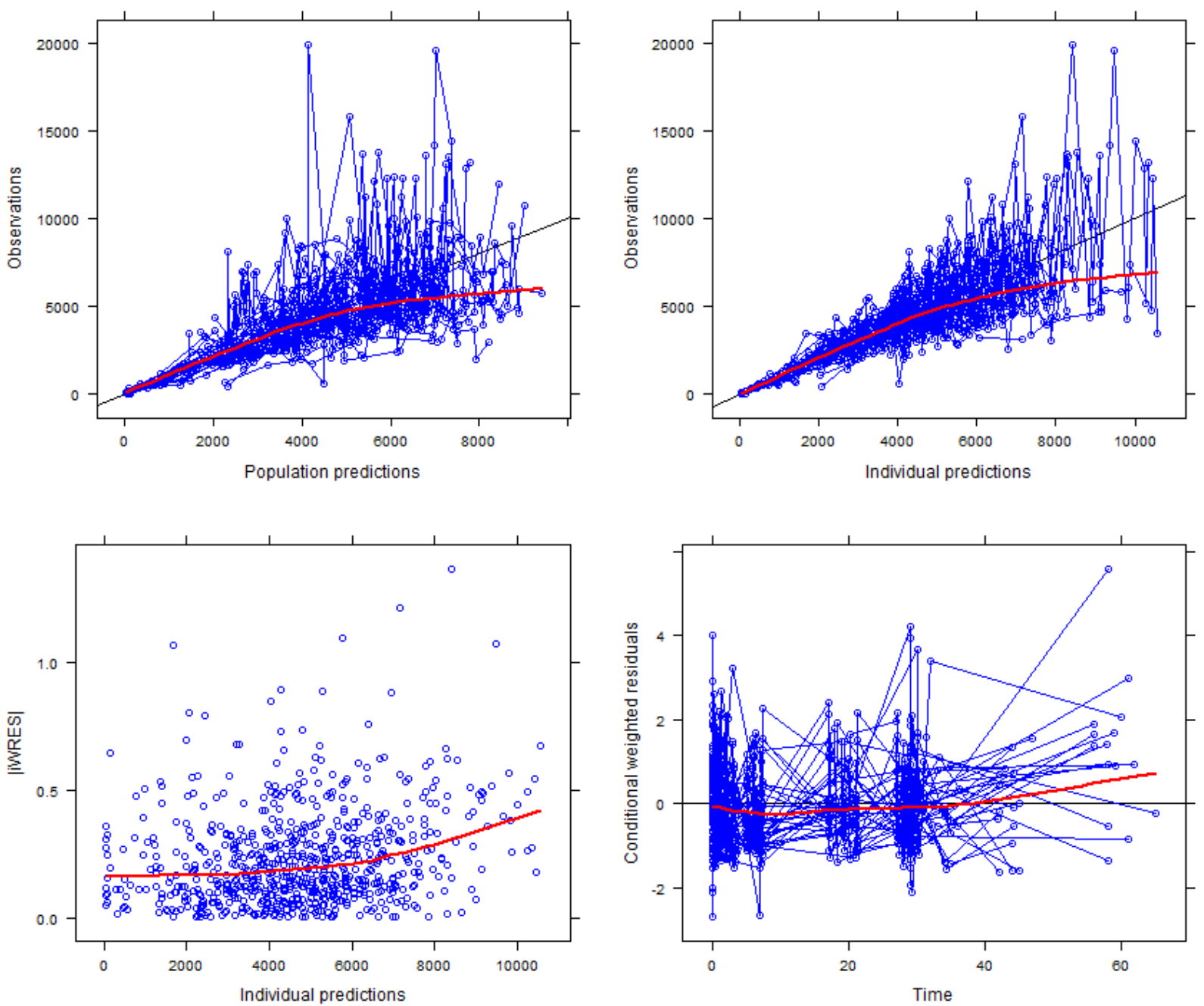

Figure E. Basic goodness of fit plots of intracellular FTC-TP model. Red line represents average values. Black line represents theoretical values. Data from the same individual are showed in blue circles and are connected by lines. |iWRES|: absolute values of individual weighted residuals. 

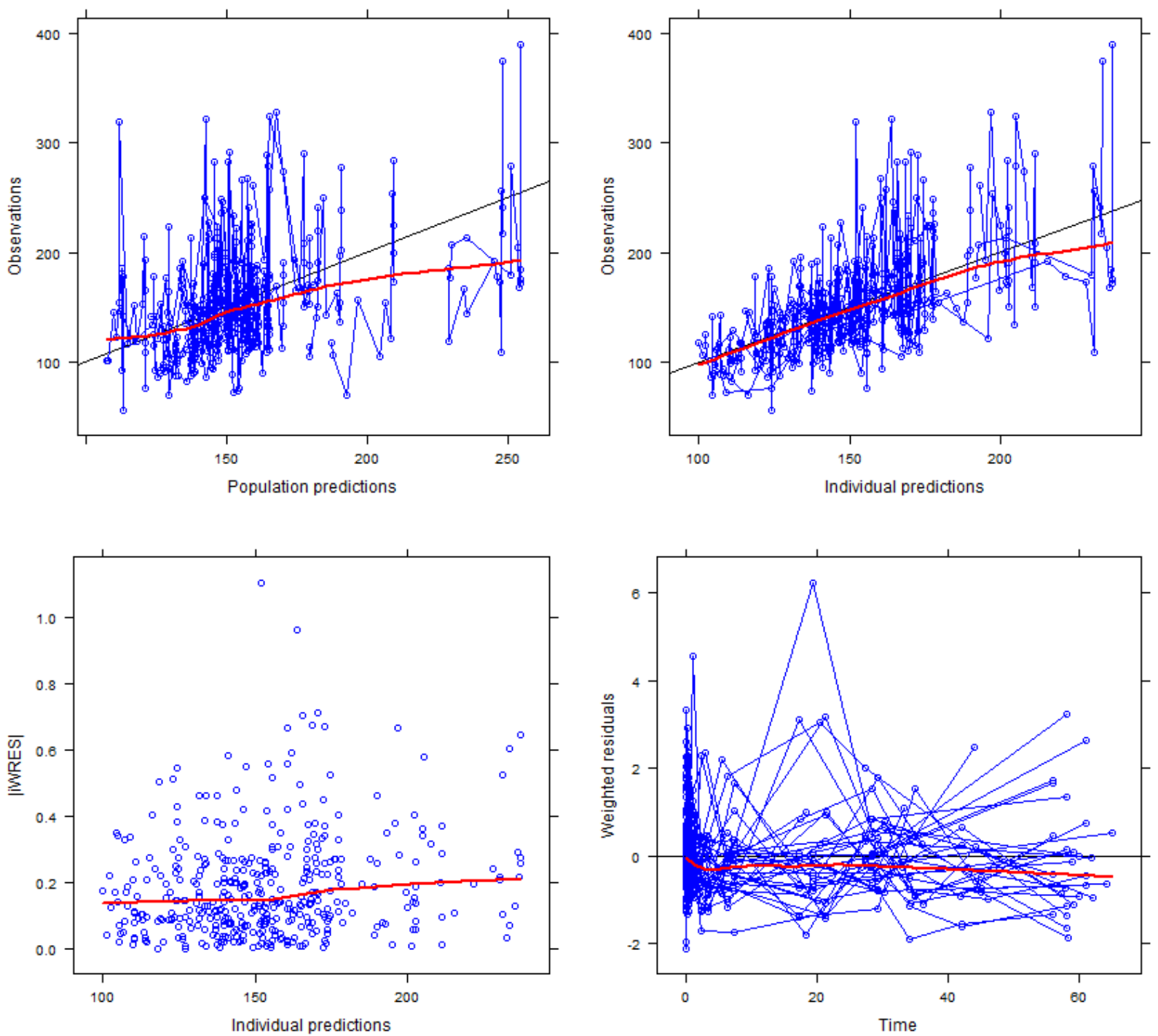

Figure F. Basic goodness of fit plots of intracellular dATP model. Red line represents average values. Black line represents theoretical values. Data from the same individual are showed in blue circles and are connected by lines. |iWRES|: absolute values of individual weighted residuals. 

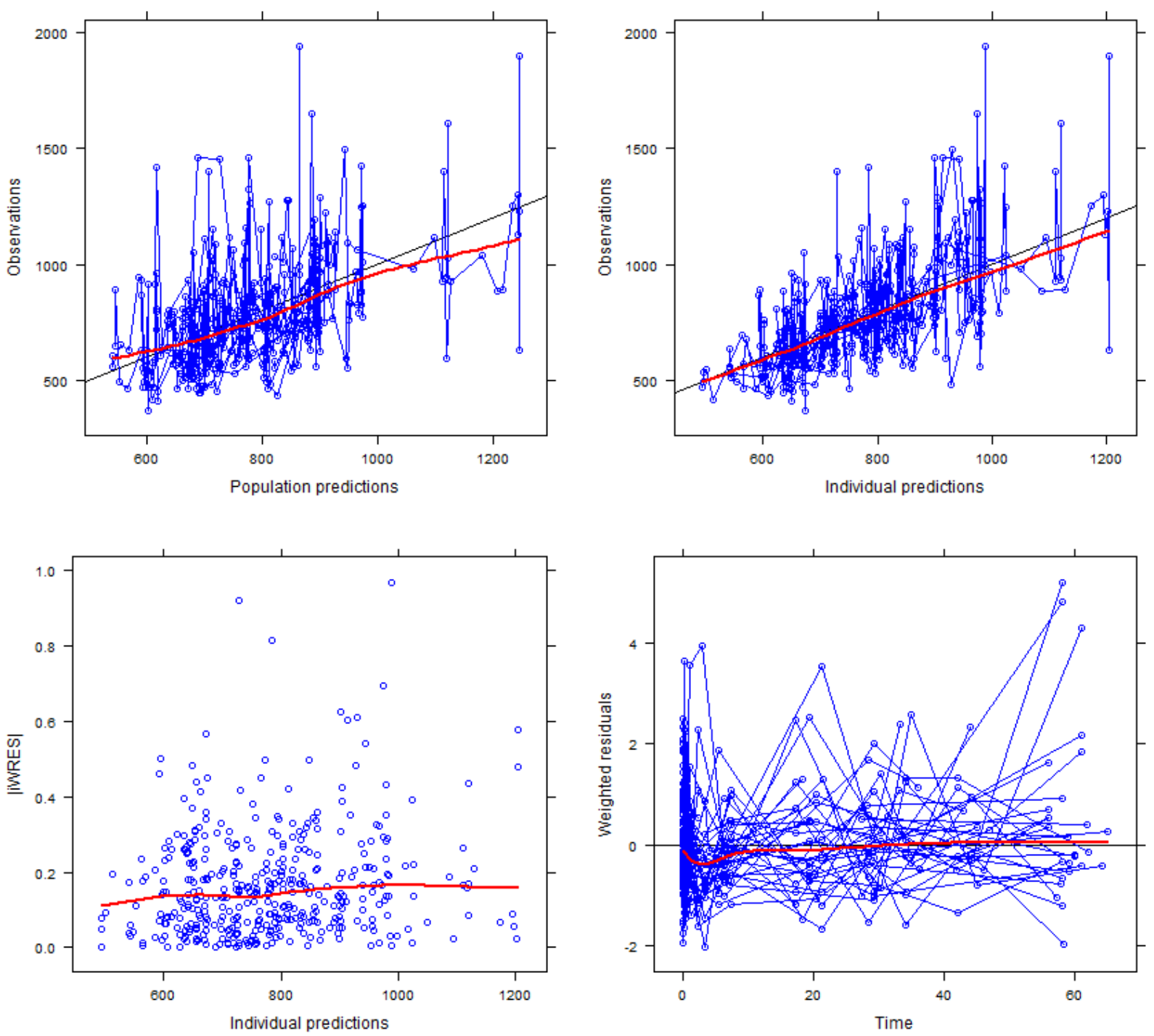

Figure G. Basic goodness of fit plots of intracellular dCTP model. Red line represents average values. Black line represents theoretical values. Data from the same individual are showed in blue circles and are connected by lines. |iWRES|: absolute values of individual weighted residuals. 

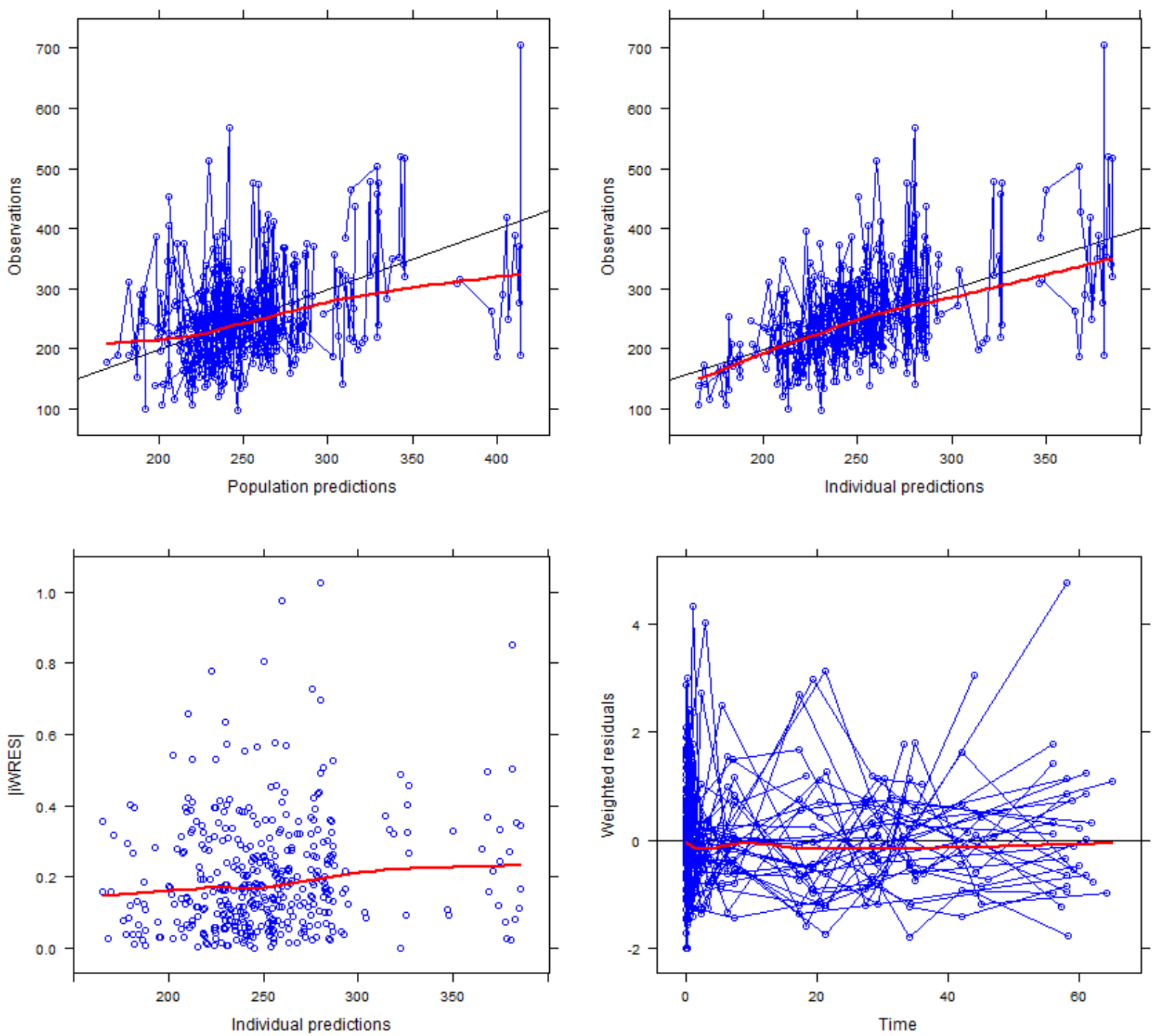

Figure H. Basic goodness of fit plots of intracellular dGTP model. Red line represents average values. Black line represents theoretical values. Data from the same individual are showed in blue circles and are connected by lines. |iWRES|: absolute values of individual weighted residuals. 

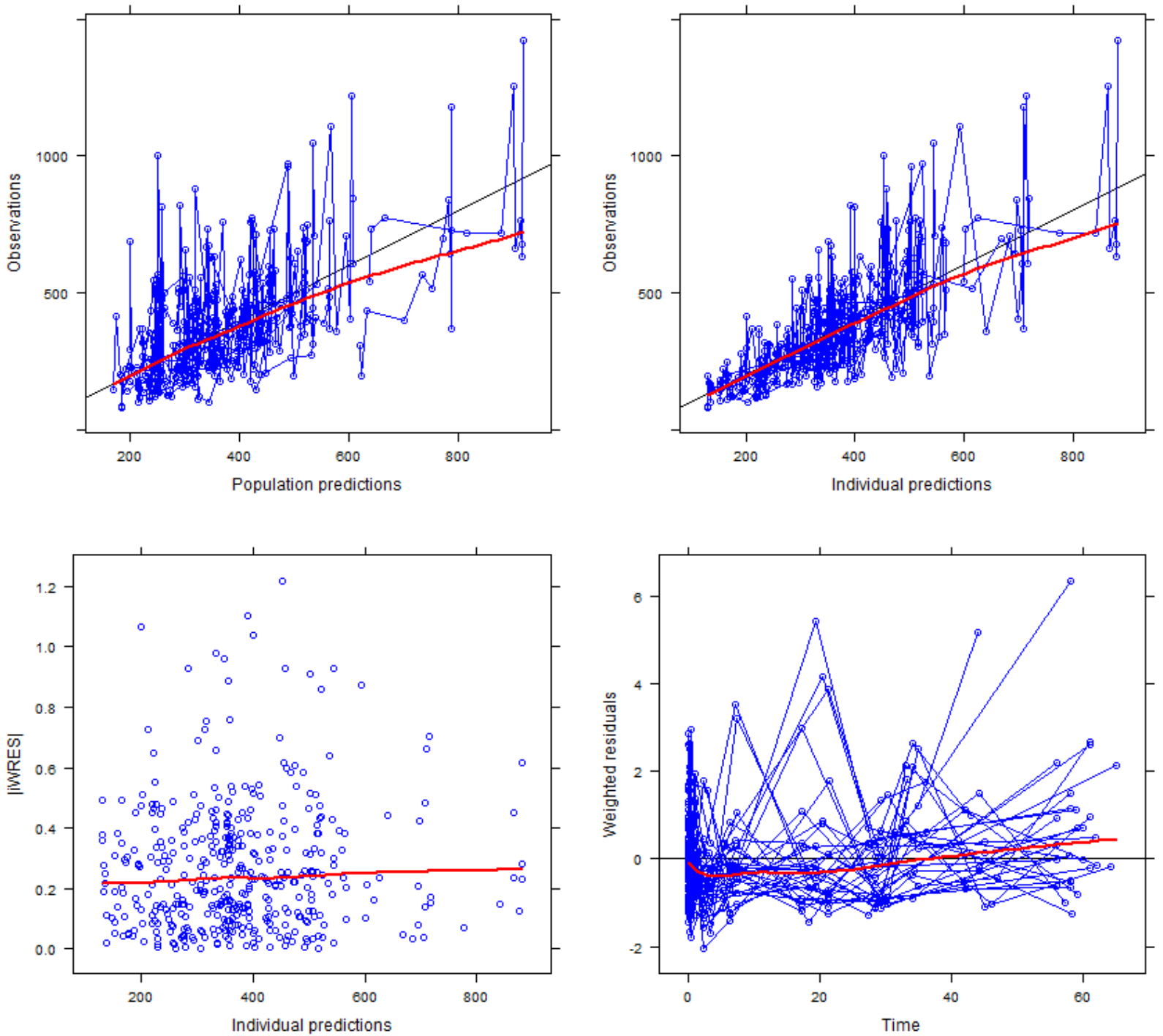

Figure I. Basic goodness of fit plots of intracellular TTP model. Red line represents average

values. Black line represents theoretical values. Data from the same individual are showed in blue circles and are connected by lines. |iWRES|: absolute values of individual weighted residuals. 
PID 1053

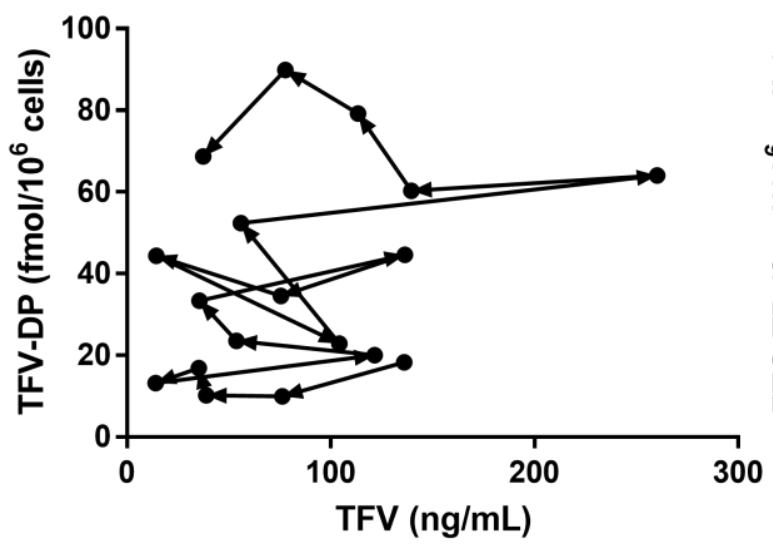

PID 1053

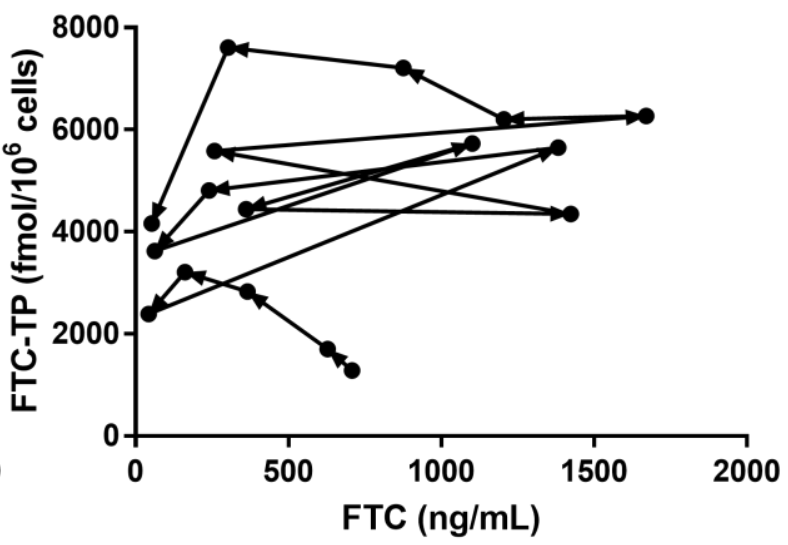

Figure J. An example of the plasma TFV/FTC vs the intracellular TFV-DP/FTC-TP "handshape"

plot. Arrows indicate the progression of time after treatment initiation. 
NONMEM code:

The NONMEM code for the modeling of concentration-time data are selectively shown below.

\section{Plasma model:}

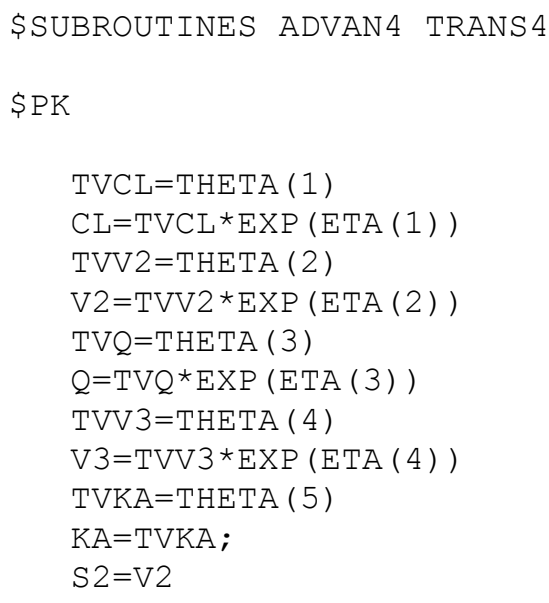

Intracellular PK link model:

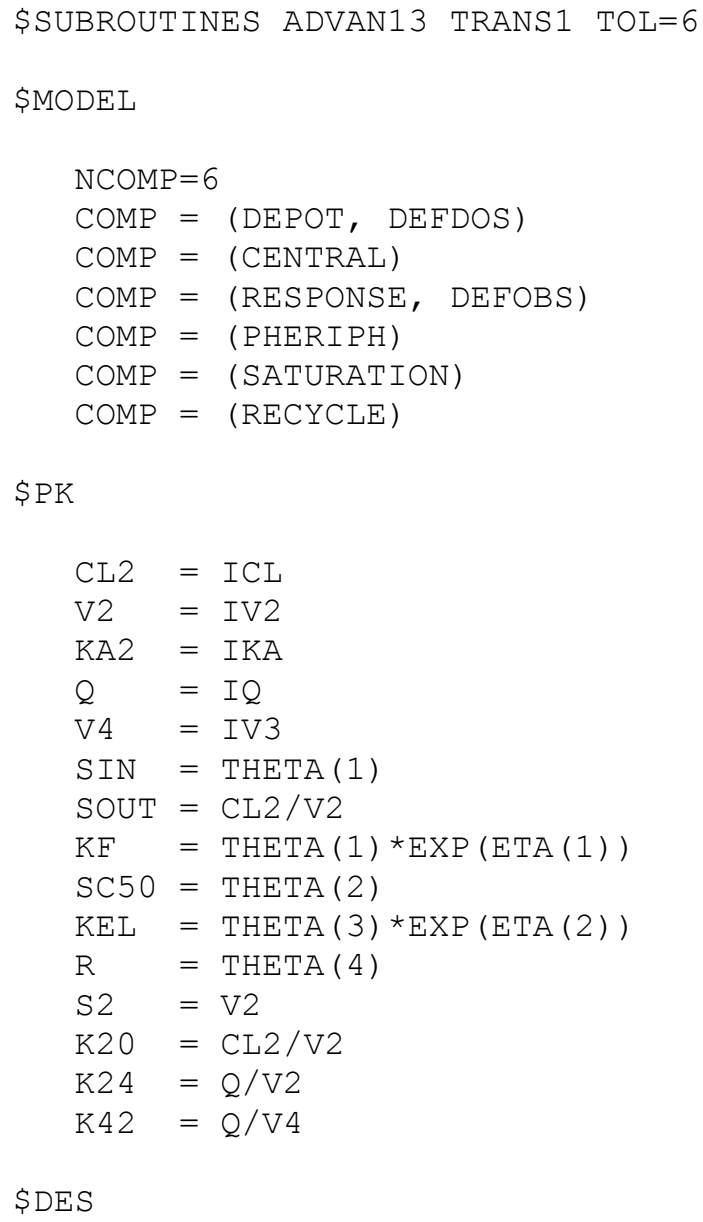




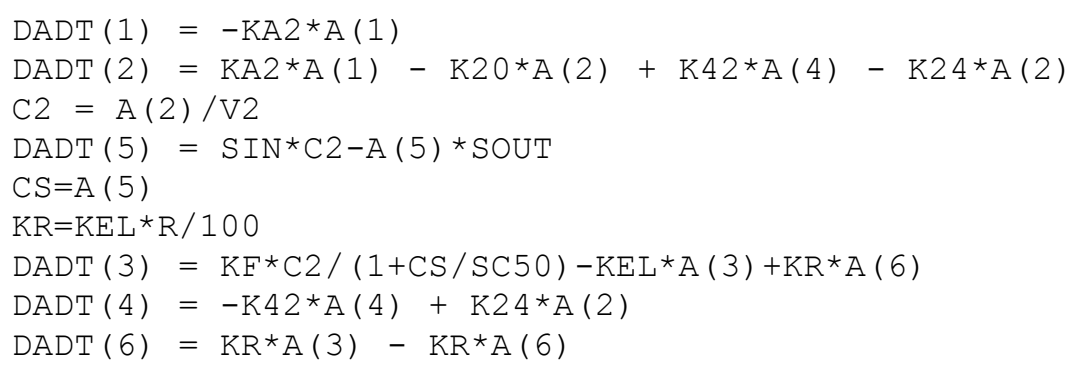

\section{Intracellular PKPD link model:}

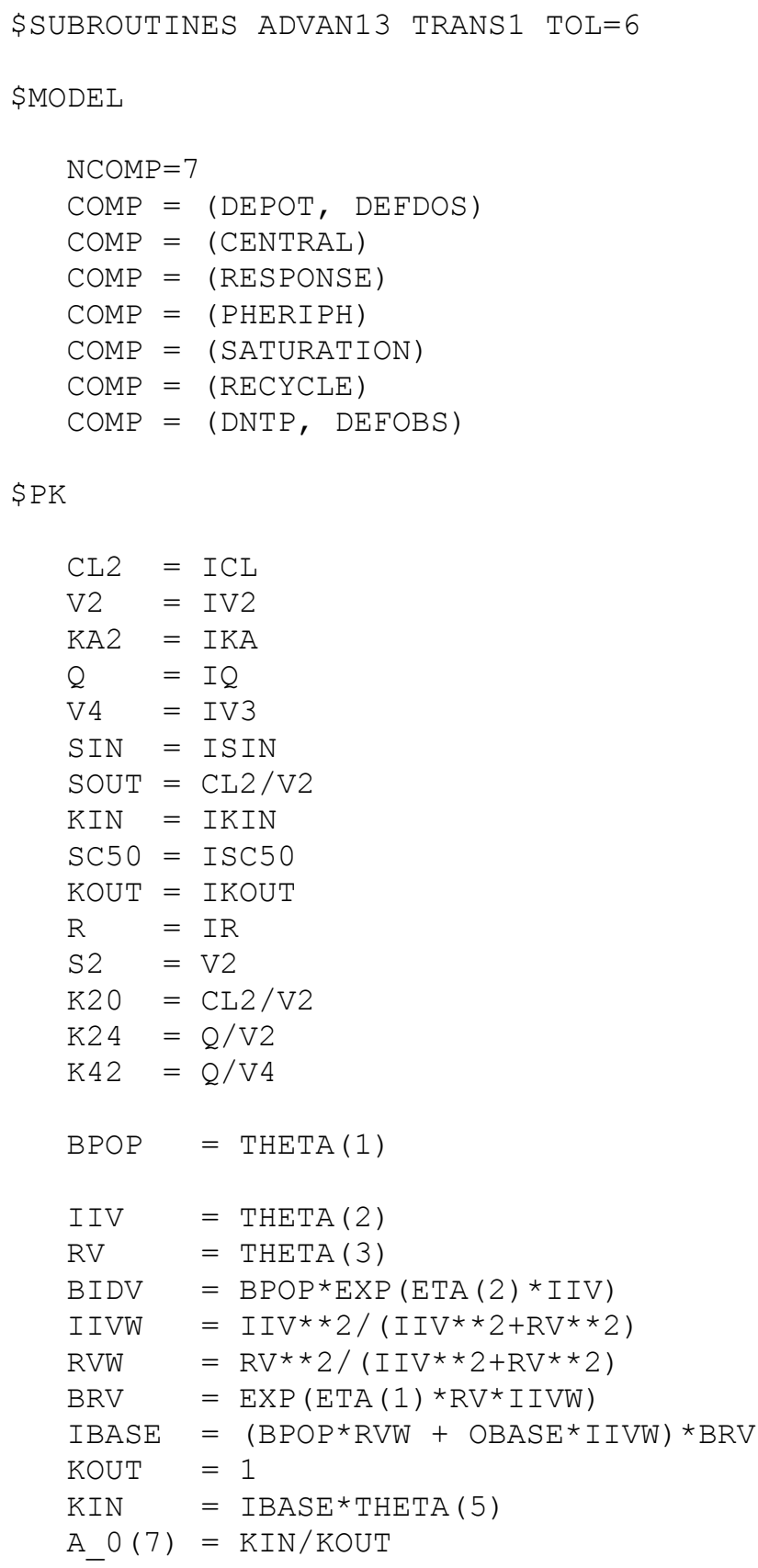


$\operatorname{EC50}=\operatorname{EXP}(\operatorname{THETA}(4)) * \operatorname{EXP}(\operatorname{ETA}(3))$

\$DES

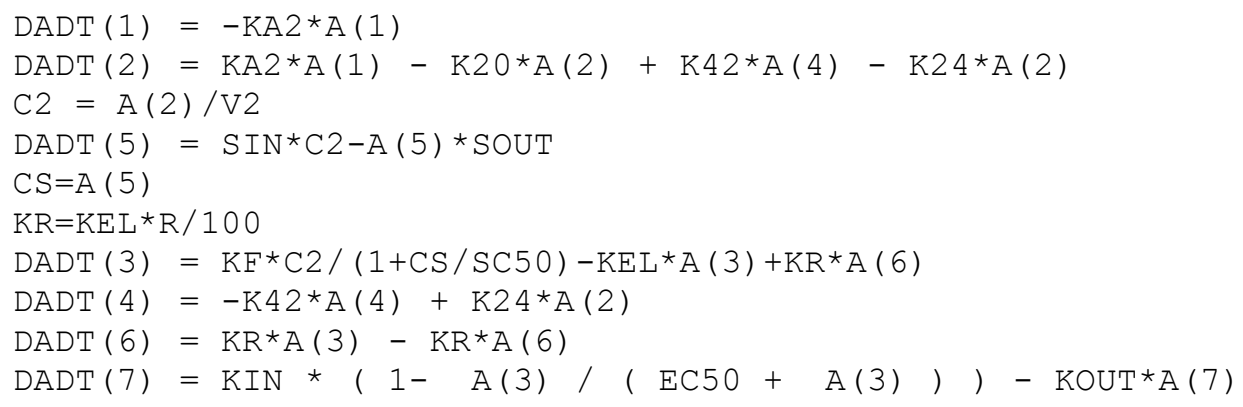

